\title{
Autonomie et dépendance des irrigants en grande hydraulique : observations de l'action organisée au Maroc et en Algérie
}

\author{
Marcel Kuper ${ }^{1}$, Mostafa Errahj ${ }^{2}$, Nicolas Faysse ${ }^{3}$, Patrick Caron ${ }^{4}$, Madjid Djebbara ${ }^{5}$, \\ Hassane Kemmoun ${ }^{6}$ \\ 1 Hydraulicien, CIRAD, UMR G-eau, Avenue Agropolis, 34398 Montpellier, France \\ 2 Socioéconomiste, ENA, Route de Hadj Kaddour, 50000 Meknès, Maroc \\ 3 Socioéconomiste, CIRAD, UMR G-eau, Avenue Agropolis, 34398 Montpellier, France \\ 4 Géographe, CIRAD, Avenue Agropolis, 34398 Montpellier, France \\ 5 Agroéconomiste, ENSA, Hacène Badi, 16200 El Harrach, Algérie \\ 6 Agronome, Cap Rural, 7 avenue Pasteur, 50000 Meknès, Maroc
}

\begin{abstract}
Il existe de nombreuses situations où l'activité agricole est administrée. C'est souvent le cas lorsque l'État cherche à valoriser des ressources productives publiques comme l'eau des barrages ou le foncier. Il impose alors, par une organisation hiérarchisée, un système technique unique, cohérent en lui-même, mais peu compatible avec la diversité des situations d'action qu'il ignore. Dans un tel contexte et sur deux exemples bien différents, les auteurs nous révèlent pourquoi et comment émergent, dans des configurations locales, des formes d'action organisée, souvent informelles. Face au redéploiement de l'État, celles-ci participent à la structuration d'une profession agricole qui, ainsi, s'émancipe. Leur analyse suppose le regard croisé de différentes disciplines sur les systèmes techniques et sociaux qui ne relève pas de la simple curiosité scientifique. Un cas exemplaire où la recherche peut accompagner les différents acteurs du développement dans la coordination de leurs actions.
\end{abstract}

La Rédaction

\section{Mots-clés :}

action organisée ; irrigants ; grande hydraulique ; recherche pluridisciplinaire ; Maghreb

\section{Keywords:} organized action; irrigators; large-scale irrigation schemes; multi-disciplinary research; North Africa

\begin{abstract}
Résumé - Constatant l'échec des associations d'irrigants et des coopératives agricoles « décrétées » dans les grands périmètres irrigués au Maghreb, il est commun d'affirmer que toute forme d'action organisée y est impossible. Pour nous, l'action organisée est, au contraire, centrale dans les recompositions en cours (disparition de certains modes de coordination hiérarchique, apparition de nouveaux acteurs, redéploiement de l'État). Nous analysons les processus qui façonnent et stabilisent les pratiques coopératives des irrigants au Maroc et en Algérie. Il existe une multitude de formes d'action organisée, souvent informelles mais rencontrant de plus en plus des formes institutionnalisées dans le domaine agricole et social. Permettant une adaptation progressive aux changements, l'action organisée sert à la fois à renforcer l'autonomie et à renégocier la dépendance des irrigants vis-à-vis de l'État.
\end{abstract}

\begin{abstract}
Autonomy and dependence of irrigators in large-scale irrigation schemes, observing organized action in Morocco and Algeria. It is conventionally stated that organized action is impossible in large-scale irrigation schemes in North Africa, at a time of profound changes in these schemes (decline of central coordination modes, appearance of new actors, diminishing role of the State), and of negative experiences with state-created cooperatives and water users' associations. We believe, on the contrary, that organized action plays a central role in these changes. We analyze the process by which formal and informal cooperative practices of irrigators are crafted, stabilized and coordinated in large-scale irrigation schemes in Morocco and Algeria. There exists a wide range of organized action, often informal but enabling
\end{abstract}

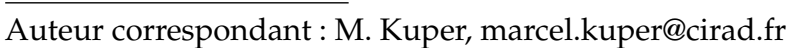


farmers to adapt progressively to change. These practices concern land and water management, purchase of inputs and marketing, as well as local development. In agriculture and social development, these cooperative practices are increasingly institutionalized. The paper shows that organized action enables irrigators to reinforce their autonomy and to renegotiate their dependence with respect to the State. Given the vigour of organized action in irrigation schemes, we recommend that its contribution to the formulation of public policies be analysed.

\section{Introduction : l'exemplarité de la grande hydraulique comme cadre d'étude}

De nombreux auteurs ont souligné l'apparente incompatibilité des agricultures familiales ${ }^{1}$ avec l'administration centralisée des périmètres irrigués de grande hydraulique (Hunt et Hunt, 1976 ; Coward, 1980 ; Lees, 1986). Le système famille-exploitation est fait « d'équilibres imbriqués, toujours précaires et évolutifs » (Osty, 1978) et les logiques et les stratégies de cette multitude d'unités familiales sont disparates et versatiles. Ces unités constituent comme une anomalie (Lees, 1986) dans un système de développement rural planifié, combinant un aménagement hydraulique et un projet de mise en valeur industrielle (coton, sucre...). Pourtant, les périmètres de grande hydraulique sont partout dans le monde constitués en majorité de ces irrigants familiaux. Selon Lees (1986), les deux parties gèrent leur apparente incompatibilité par des arrangements informels ${ }^{2}$ pour obtenir une flexibilité opérationnelle. Ces arrangements permettent, d'une part, à un système de régulation rigide (règles standardisées et peu évolutives) de se perpétuer et, d'autre part, aux irrigants de "survivre » au sein de tels périmètres.

Le modèle de grande hydraulique fut conçu et mis en place dans différents continents à partir de la deuxième partie du XIXe siècle dans un contexte de coordination hiérarchique très prégnant (Diemer et Slabbers, 1992). L'État aménageur ne dirigeait alors pas seulement la mobilisation et la distribution de l'eau, il intervenait fortement dans les orientations des exploitations agricoles, dans la conduite de l'exploitation et dans la transformation et la commercialisation des productions. Partout, ce modèle fut soumis à de fortes perturbations depuis le début des années 1980 par une libéralisation politique et économique : émancipation des irrigants, apparition de nouveaux

\footnotetext{
1 Selon Mercoiret (2005, p. 19), les agricultures familiales se caractérisent "par la prédominance du travail des membres de la famille et une organisation familiale du processus de production, [...] l'existence d'une articulation étroite entre production, consommation familiale et reproduction du groupe domestique».

2 Elle les définit comme des «actions de contournement des règles ou procédures formelles. À travers ces arrangements, les participants peuvent se comporter comme s'ils étaient suffisamment en conformité avec les règles afin de ne pas perdre les avantages de leur participation, tout en s'affranchissant de certaines conditions $»$.
}

acteurs et redéfinition du rôle de l'État (Egg et Deme, 2002). D'un mode de coordination hiérarchique, il fallait passer à des modes de coordination impliquant davantage les « usagers» (Johnson et al., 1995), puis faisant intervenir des mécanismes du marché avec la libéralisation des assolements, le démantèlement de filières intégrées et la libéralisation des marchés agricoles.

Les exploitants des unités familiales au sein des périmètres de grande hydraulique se retrouvent alors dans une situation paradoxale. D'une part, ils continuent de dépendre d'un organisme gestionnaire de l'eau, le plus souvent étatique, et sont appelés à participer à des associations d'usagers de l'eau et à des coopératives agricoles « décrétées » par l'État. D'autre part, le désengagement de l'État conduit à la recherche de nouvelles autonomies et, pour cela, à de nouvelles coordinations, qu'il s'agisse d'être présent sur des marchés évolutifs, de mobiliser de nouvelles ressources ou encore de mettre en œuvre de nouvelles manières de produire.

Nous posons l'hypothèse selon laquelle les arrangements informels décrits par Lees (1986) jouent un rôle important dans les recompositions en cours, permettant aux exploitants de s'adapter à des changements profonds et rapides auxquels ils ne peuvent faire face individuellement. Nous prolongeons cette hypothèse en postulant que le contexte actuel de libéralisation politique et économique offre des opportunités de stabilisation et de formalisation des arrangements vers des formes d'action organisée ${ }^{3}$ dans le sens attribué par Friedberg (1993). À côté des formes préexistantes, mais souvent occultées, d'arrangements informels dans un contexte de coordination hiérarchique, on observe aujourd'hui des actions plus formalisées induites par la modification de l'action et de la posture de l'État. Cet article propose d'analyser le processus par lequel sont façonnées, stabilisées et coordonnées les pratiques coopératives (formelles et informelles) impliquant principalement les agriculteurs au sein des périmètres de grande hydraulique au Maroc et en Algérie. Nous employons le terme «action organisée » de Friedberg (2005) pour désigner l'ensemble des pratiques coopératives, qu'elles soient formelles ou informelles. Nous formulons l'hypothèse que l'action organisée des irrigants sert à la fois à renforcer leur autonomie et à renégocier leur dépendance vis-à-vis de l'État.

\footnotetext{
3 « Processus d'organisation par lesquels sont façonnés, stabilisés et coordonnés les comportements et les interactions stratégiques d'un certain nombre d'acteurs dont l'interdépendance rend la coopération indispensable... » (p. 21).
} 
Constatant l'échec de certaines formes d'action organisée « décrétée » dans ces périmètres, il est commun d'affirmer que toute forme d'action organisée est impossible, que les agriculteurs sont bien trop individualistes pour savoir et pouvoir s'engager collectivement. Il devient ainsi facile à la fois pour les services administratifs et pour les agriculteurs de justifier l'échec des actions entreprises et l'inaction, à un moment où le changement de régime d'action est bien déroutant et remet en cause les justifications et les identités professionnelles de chacun. Il existe un véritable enjeu à démontrer que l'action organisée existe, à la repérer, l'identifier, l'interpréter et la qualifier pour dépasser les discours convenus d'immobilisme. Plus généralement, la problématique de la grande hydraulique semble exemplaire et particulièrement illustrative des processus en jeu dans l'évolution, en milieu paysan, de l'action organisée et, par voie de conséquence, dans l'interrogation du rôle de l'État et de l'action publique.

Dans ce qui suit, nous clarifions d'abord les choix méthodologiques que nous avons faits pour aborder l'action organisée en grande hydraulique, avant de présenter deux études de cas $^{4}$. Le premier cas illustre la stabilisation de l'action organisée au Maroc; le deuxième mobilise des observations réalisées en Algérie, dans un contexte radicalement différent de par la conception de l'action de l'État et qui se caractérise par la prépondérance d'actions informelles. Dans la discussion, nous revenons sur notre hypothèse que l'action organisée sert à la fois à renforcer l'autonomie et à renégocier la dépendance des irrigants par rapport à l'État.

\section{Des choix méthodologiques pour aborder l'action organisée en grande hydraulique}

L'approche suivie pour analyser les différentes formes d'action organisée des agriculteurs est inspirée du « retournement de perspective » proposé par Jollivet (1992). $\mathrm{Au}$ lieu d'examiner les raisons d'échec et les dysfonctionnements des structures de gestion de l'eau ou de production agricole "décrétées » par l'État, il est plus opportun de repérer et de qualifier des pratiques coopératives fonctionnelles, qu'elles soient formalisées ou pas, pour aboutir éventuellement à des recommandations de reformulation des politiques publiques et contribuer à la création d'espaces de négociation.

$\mathrm{Au}$ Maroc comme en Algérie, les associations d'irrigants et les coopératives de production étaient dans le passé l'émanation de l'administration (depuis les

\footnotetext{
${ }^{4}$ L'article est basé sur une série de recherches menées par une équipe pluridisciplinaire dans le cadre du projet SIRMA (Économies d'eau en systèmes irrigués au Maghreb) : cf. http://www.eau-sirma.net.
}

indépendances jusqu'à l'avènement des libéralisations politique et économique). Ces organisations ont longtemps souffert d'une image très négative (Desrues, 2005) : manque de représentativité, monopole de la représentation (une seule organisation par filière), manque de démocratie interne, ingérence des services de l'État... En conséquence, dans les deux contextes, se sont développées chez les agriculteurs des attitudes négatives vis-à-vis de l'action organisée, qu'elle concerne l'irrigation ou la production agricole. Elles se traduisent par un rejet des structures collectives, qui restent des " coquilles vides ", ou par le détournement de leurs fonctions. Dans les deux cas, pour accéder aux ressources, affronter le marché ou contribuer au développement local, les agriculteurs procèdent par des arrangements à petites échelles et dans le cadre de réseaux de proximité. Ces arrangements sont pour nous un objet de recherche qui nous éclaire sur les opportunités de leur stabilisation et leur formalisation, ce qui n'est autre que l'action organisée (Friedberg, 1993; Ostrom, 1992).

Nos premières observations au Maroc montrent l'émergence de coopératives et d'associations jugées fonctionnelles par les irrigants, principalement à l'échelle locale, jouant un rôle économique et social important (Kemmoun et al., 2004). La reconquête et l'appropriation de ces structures témoignent d'une volonté des irrigants de prendre en main leur destin. Shah (1995) montre comment, en Inde, des organisations professionnelles structurent les filières agricoles, prennent en charge la transformation et la commercialisation des produits agricoles, la défense de la profession, voire le développement local au profit de l'agriculture familiale. Nous avons fait le pari que ces organisations constituent des lieux privilégiés pour observer « des dynamiques sociales à l'interface des enjeux locaux et nationaux » (Jacob et Lavigne Delville, 1994, p. 14), même si l'action organisée en milieu rural ne se limite pas à ces organisations.

En théorisant les différents modes de coordination à propos de la gestion de biens communs, des auteurs comme Coward (1980) et Ostrom (1992) ont influencé les politiques publiques de l'irrigation. Ils ont conseillé une implication plus importante des agriculteurs dans la gestion de l'eau au sein de la grande hydraulique. Dans la pratique, la création des associations d'usagers de l'eau agricole (AUEA) était souvent décrétée par l'État et mise en œuvre par l'administration hydraulique. Aujourd'hui, les AUEA sont peu fonctionnelles (Mollinga et Bolding, 2004). C'est le cas au Maroc et en Algérie, où l'État garde largement le contrôle de l'allocation et la distribution de l'eau des barrages, malgré le rôle consultatif des AUEA. Les agriculteurs maintiennent une certaine flexibilité opérationnelle à travers des arrangements de proximité (échange de tour d'eau...). Ils ont obtenu une marge de manœuvre bien plus importante encore en ayant recours à la nappe par pompage, individuellement ou à travers 
des arrangements de proximité. Concernant la gestion de l'eau, nous avons donc choisi une entrée par l'analyse des arrangements d'accès à l'eau souterraine.

Nous avons privilégié les enquêtes (guide d'entretien, questionnaire, récits de vie) sur les différentes formes d'action organisée à différents niveaux d'analyse (individu, exploitation agricole, groupes locaux). L'observation des pratiques coopératives nous a permis de mettre en perspective les discours recueillis. Nous avons réinterprété l'évolution de l'action organisée au regard des trajectoires de développement local (Caron, 1998). La méthodologie s'appuie sur la réalisation d'enquêtes monographiques portant sur les transformations d'un espace particulier.

\section{Les coopératives laitières, une illustration d'action organisée en grande hydraulique au Maroc}

Au Maroc, où la politique de grande hydraulique fut relancée avec vigueur après l'indépendance, l'État promulgua en 1969 le Code des investissements agricoles pour encourager les agriculteurs à moderniser leurs exploitations et augmenter la productivité. L'État était responsable aussi bien des équipements externes à l'unité de production (remembrement, adduction d'eau) que des équipements internes (défrichement, drainage, nivellement des terres); les agriculteurs avaient accès à des intrants subventionnés, des crédits et des prêts de machines agricoles (Popp, 1984). En contrepartie, ils devaient payer une redevance d'eau et suivre les assolements fixés par l'administration, se trouvant ainsi dans un « réseau serré d'obligations » (Bouderbala, 1977). L'avènement du programme d'ajustement structurel, au début des années 1980, a conduit progressivement au retrait de l'État des services à caractère commercial, à la libéralisation des assolements, à la privatisation des unités agroalimentaires et à la constitution d'AUEA.

En prenant comme support la caractérisation de l'action organisée en zone irriguée (et non nécessairement à propos de l'irrigation elle-même), la situation retenue ici illustre la diversité des formes d'émergence de cette action organisée au regard des transformations des modes d'action de l'État. Elle montre, à travers le renouvellement des formes coopératives en production laitière, comment les producteurs s'organisent pour pallier les défaillances d'initiatives autrefois fortement pilotées par les services de l'État. Le lait a connu un essor important en périmètre irrigué au Maroc, touchant un très grand nombre d'agriculteurs familiaux. Les fourrages y constituent souvent plus de $25 \%$ des assolements et les périmètres irrigués assurent près de $45 \%$ de la production laitière (Sraïri et al., 2007).

\section{Pour le développement local : la recherche d'autonomie d'une coopérative laitière}

Menée par un groupe de jeunes, une communauté dans le périmètre du Tadla au Maroc décide, à la fin des années 1990, de créer sa propre coopérative laitière, en se séparant de la coopérative «mère ». Le rejet de l'ancienne coopérative était lié à son fonctionnement jugé « opaque » (contrôle des comptes, déroulement des assemblées générales). La communauté estimait en outre ne pas bénéficier de ses programmes sociaux (réfection de pistes, notamment). Considérant que la filière avait besoin de solides coopératives de taille importante, les autorités et l'industriel de la zone ont découragé cette initiative. La détermination de la communauté, l'habileté de ses leaders et l'ouverture politique ont néanmoins permis la création d'une nouvelle coopérative en 2001. Solidement installée, la coopérative livre en 2006-2007 près de 550000 litres de lait par an à l'industriel pour environ 70 adhérents et 30 occasionnels. Le chiffre d'affaires du lait représente 1,8 million de dirhams (environ $160000 €$ ). Chaque adhérent livre en moyenne 6000 litres par an pour un peu moins de 4 vaches laitières par exploitation.

La coopérative a pour stratégie d'augmenter la productivité des vaches et d'améliorer la qualité du lait afin de bénéficier des primes de qualité octroyées par l'industriel et attribuées à la coopérative : elle a reçu en moyenne une prime de 20 centimes/litre contre seulement 8 pour la coopérative mère en 2004-2005 (Oudin, 2006). Au lieu de redistribuer ces primes aux adhérents, la coopérative obtient un premier niveau d'autonomie en créant un fonds financier. Les primes, d'un montant annuel de 150000 dirhams (environ $14000 €$ ), constituent ainsi le moyen pour la coopérative d'investir dans l'amélioration du dispositif de collecte (bâtiment, personnel), mais aussi dans des services annexes fidélisant les adhérents. En 2008, la coopérative a recruté un technicien d'élevage et mis en place un programme de formation pour les adhérents afin d'augmenter la productivité laitière. En pilotant un programme technique, autrefois géré par les services de l'État, la coopérative vise un deuxième niveau d'autonomie. La diversification des services lui permet de répondre aux attentes de la communauté et de renforcer son ancrage local. Au-delà de la fonction économique, liée à la promotion de la production laitière, la coopérative intervient dans l'amélioration des conditions de vie en participant à l'identification et au financement de projets sociaux. Elle intervient ainsi dans la sphère publique, créant de fait un troisième niveau $\mathrm{d}^{\prime}$ autonomie. Ces projets sont pilotés par une association de développement dans le douar, étroitement liée à la coopérative laitière, mais bien distincte par une séparation des comptes bancaires.

L'association a été créée une année après le démarrage de la coopérative. Elle intervient dans l'école publique, elle a créé une infirmerie, gère une ambulance et a négocié 
la mise à disposition d'un médecin de l'hôpital public à temps partiel. Elle est aussi intervenue dans les négociations pour l'approvisionnement en eau potable des habitants et la réfection des pistes. Les leaders de cette association sont en grande partie les mêmes qui sont actifs dans le pilotage de la coopérative laitière. La synergie créée entre ces deux structures témoigne d'une volonté explicite de la communauté de prendre en main sa destinée. Les liens resserrés entre la coopérative laitière et l'association de développement - on pourrait parler d'ingénierie institutionnelle - assurent des capacités de réflexion, de négociation et d'action importantes. La coopérative fournit l'outil économique à travers une contribution annuelle à l'association et une mise à disposition de ses locaux (salle de réunion, infirmerie, garage...), mais constitue aussi un lieu de rencontre et de discussion grâce au passage quotidien pour les livraisons de lait. L'association, quant à elle, est sollicitée pour répondre à des problèmes d'ordre social dans le douar. Ses services sont destinés à l'ensemble de la population du douar (et non pas aux seuls adhérents de la coopérative laitière). La communauté s'est ainsi approprié à la fois l'outil économique et l'outil de développement social.

Oudin (2006) montre que la presque totalité des coopératives laitières du périmètre du Tadla offrent des services annexes, dans les domaines économiques et sociaux, à la collecte de lait. Une croissance de la production laitière (91 millions de litres de lait collectés au Tadla en 2005-2006) est conjuguée à une intervention importante dans le développement local des communautés concernées. Même si ces coopératives rencontrent des difficultés (jeux de pouvoir internes, parfois un manque de rigueur dans la gestion...), elles constituent incontestablement des plateformes de coordination et de négociation pour les communautés. Ce constat est aussi valable pour d'autres périmètres de grande hydraulique au Maroc (Kemmoun et al., 2004).

\section{Une illustration d'arrangements de proximité en Algérie}

En Algérie, l'État se voulait dès l'indépendance l'acteur principal d'un ambitieux projet de développement (Guillermou, 1994), en regroupant 22000 fermes coloniales d'une superficie de 2,3 millions d'hectares en 2000 domaines agricoles et en nationalisant 1,2 million d'hectares de grands privés (Jouve, 1999). L'aide publique s'est presque exclusivement orientée vers le domaine agricole public : approvisionnement et subvention d'intrants, organisation et contrôle de la production et de la commercialisation, affectation d'ingénieurs au sein des domaines (Jouve, op . cit. ; Mesli, 2007). Dans un contexte de crise économique amplifiée par un climat politique difficile (Bessaoud, 2006), le secteur agricole public a connu un retrait marqué de l'État depuis la dernière réforme en 1987 : dissolution des domaines, constitution d'exploitations agricoles collectives (EAC) ou individuelles (EAI), désengagement de l'État de l'acte de gestion de l'exploitation agricole, restitution des terres nationalisées. Ce n'est qu'en 2000 qu'une relance de l'agriculture fut mise en œuvre à travers le programme national de développement agricole, pour un montant de 3,5 milliards d'euros et profitant à $220000 \mathrm{EAC}$ et EAI (ibid.).

\section{Pour «l'accès aux ressources »: les arrangements de proximité dans la Mitidja}

Notre investigation a concerné la commune de Mouzaïa, située à $60 \mathrm{~km}$ au sud-ouest d'Alger dans la plaine de la Mitidja. À l'image de toute la plaine, Mouzaïa a connu une histoire agraire riche, attirant tôt les convoitises de la colonisation (Chaulet, 1984). Dans la première tranche d'irrigation du périmètre de la Mitidja ouest, 5600 hectares à Mouzaiia ont été équipés dans les années 1980. Les agriculteurs ont largement recours aux eaux souterraines, en raison notamment du dysfonctionnement du barrage.

Le retrait rapide de l'État depuis 1987 a eu pour conséquence un bouleversement du paysage agraire dans le domaine public, découlant, au fil du temps, d'une prolifération d'arrangements de proximité entre les attributaires des EAC de Mouzaïa. Chaque EAC regroupe 5 à 15 anciens ouvriers et techniciens des domaines agricoles pour environ 15 à 50 hectares, qu'ils sont censés travailler ensemble. Les arrangements concernent le foncier, mais aussi l'accès à l'eau et d'autres activités agricoles (gestion des vergers d'agrumes, notamment). Dans un contexte d'attitudes négatives vis-à-vis des organisations professionnelles, il n'est finalement pas surprenant de constater que nombreux sont les arrangements de proximité et que l'action organisée des irrigants se construit essentiellement hors de l'action publique et des cadres coopératifs « décrétés ». Il s'ensuit un essor agraire non négligeable, avec 1200 hectares de nouvelles plantations d'agrumes et de rosacées (pêche, pomme, notamment) et plus de 800 hectares de maraîchage de plein champ et sous serre (Imache, 2008).

Les arrangements autour du foncier concernent le partage informel de la terre par les attributaires des EAC et la location de terres; plus rarement, on observe aussi la vente des terres. Ce sont des pratiques courantes, alors que le foncier est la propriété de l'État et que tout partage, vente ou location est légalement interdit. Le partage du foncier, suite à des mésententes entre attributaires, est adapté aux situations spécifiques de chaque EAC; la présence d'un verger, l'accès à un forage et le partage du matériel agricole déterminent les clés de répartition. Ce partage informel est très répandu dans le périmètre : dans la commune de Mouzaïa, 73 \% des 182 EAC sont 
" éclatées ", avec un partage de facto de la terre entre attributaires (ibid.). Dans la plupart des cas, le partage n'aboutit pas à des exploitations individuelles, mais à des petits collectifs de 2 à 7 membres ayant souvent des liens de parenté (frères, cousins). Il est intéressant de noter que «l'éclatement » n'empêche pas les attributaires des EAC de continuer à s'entraider (échanges de service pour la main-d'œuvre et le matériel agricole, par exemple), ni de faire des investissements communs. Nous avons pu observer, par exemple, l'installation d'un transformateur électrique pour des pompes immergées, commun à 4 EAC «éclatées ». Cela n'empêche pas non plus les attributaires d'une EAC « éclatée » de constituer collectivement des dossiers de subvention de l'État (systèmes de goutte-àgoutte, plantations). Les attributaires montrent ainsi un sens pratique et restent « stratèges dans l'utilisation des offres étatiques », comme l'avait déjà remarqué Chaulet (1984).

La location de terres est également très répandue. Une enquête effectuée à Mouzaïa en 2006 a montré que, sur 182 EAC, plus du tiers étaient concernées. Pour des locations à long terme (supérieur à 5 ans), il existe généralement un acte notarié pour protéger les deux parties, même si le document n'a que peu de valeur juridique. Le locataire est libre de choisir l'assolement et peut même procéder à la plantation de vergers d'agrumes ou de rosacées. Pour les locations à court terme - sur une campagne agricole -, il n'existe pas d'acte notarié. La culture est précisée avant de conclure l'arrangement et concerne des cultures maraîchères (sous serre ou de plein champ) ou fourragères. Le locataire s'assure d'abord d'un accès à l'eau avant de prendre en location la terre. La présence d'un forage permet un prix de location qui peut atteindre six fois la valeur d'une location sans eau. Imache (2008) montre que les locataires contribuent ainsi largement aux dynamiques agraires actuelles et sont porteurs d'innovation (introduction de nouvelles cultures, itinéraires techniques, systèmes d'irrigation).

Les arrangements autour de l'eau concernent l'eau du barrage, mais surtout celle des forages. En l'absence d'un approvisionnement régulier du barrage, les forages « illicites » (280 forages pour 182 EAC à Mouzaïa) portent largement les dynamiques agraires actuelles (ibid.). De nombreux arrangements se sont greffés sur ces forages pour fournir de l'eau au plus grand nombre d'irrigants, y compris aux locataires informels, procurant ainsi une certaine autonomie par rapport à l'eau publique. Les arrangements autour des forages sont de plusieurs types : (i) investissement collectif (forage, moteur, pompe); (ii) exploitation collective d'un forage hérité d'avant la réforme de 1987; (iii) transactions ponctuelles. Les deux premiers arrangements sont par nature de longue durée et connaissent souvent des perturbations considérables suivant l'entente des attributaires de l'EAC concernée. La gestion du tour d'eau en période de pénurie et la contribution aux frais d'entretien fragilisent assez souvent ces arrangements. Les transactions concernent à la fois des actes de solidarité et de bon voisinage (parents, voisins) et la vente d'eau, surtout pour des locataires. Ces arrangements sont ponctuels et n'excèdent que rarement une campagne agricole.

\section{Discussion : regards croisés sur ces illustrations}

Nous avons vu à travers ces illustrations que les mythes d'agriculteurs immobiles et trop individualistes pour faire évoluer collectivement leurs conditions de vie et de travail rendent mal compte des dynamiques de l'action organisée que nous avons observées. L'action organisée existe bel et bien en grande hydraulique, même si elle rencontre des difficultés.

La portée et la vigueur de l'action organisée deviennent ainsi des marqueurs d'un changement du régime $\mathrm{d}$ 'action. Alors que les irrigants, acteurs de l'action organisée, cherchent d'abord à minimiser les risques (mutualisation, transfert) et à renforcer leur autonomie, en sortant d'un contexte de coordination hiérarchique, on observe, paradoxalement, qu'étant plus forts et plus organisés, ils cherchent en même temps à renégocier leurs relations de dépendance envers l'État, à travers le dialogue. Parfois, ils ne réclament qu'un soutien moral de l'État. Mais n'est-ce pas, en fait, une demande de reconnaissance, une envie d'affirmation de la part d'un groupe professionnel que l'administration a tendance à vouloir maintenir dans une position d'usager de service ou de cible pour des programmes de vulgarisation? La coopérative de lait, pour sa part, négocie des contributions de l'État à ses programmes économiques et sociaux, telle la mise à disposition d'un médecin. Les locataires de la Mitidja, quant à eux, cherchent à obtenir la carte fellah, qui leur permettrait d'être reconnus comme exploitants agricoles à part entière (Imache et al., à paraître). Nous estimons que l'action organisée est au centre des recompositions en grande hydraulique. Il nous semble qu'à travers ces différentes formes d'action organisée, une émancipation et une professionnalisation de l'agriculture familiale sont bien en cours.

Devant les multiples contraintes d'accès à l'eau et au foncier, et les relations difficiles avec les marchés, les agriculteurs continuent à fonctionner via des arrangements informels, selon l'hypothèse de Lees (1986). C'est particulièrement vrai pour les locataires de la Mitidja, porteurs de véritables dynamiques agricoles mais sur un foncier non sécurisé et utilisant l'eau de forages «illicites ». On y trouve une forte capacité à «mobiliser l'illicite pour trouver un autre système d'ordre » (J.-P. Billaud, comm. pers.). Le manque de reconnaissance des locataires et le caractère informel de l'action organisée pour l'accès aux 
ressources (eau, foncier) pourraient fragiliser les dynamiques agraires actuelles. L'État algérien réfléchit à la mise en place d'une nouvelle réglementation foncière, notamment pour les EAC. L'enjeu sera de sécuriser les agriculteurs exploitant la terre, ce qui passe probablement par la reconnaissance de l'ensemble des irrigants présents (y compris les locataires) afin de construire sur les dynamiques existantes.

Mais nous pouvons aller plus loin que Lees (1986), en constatant qu'en grande hydraulique, au Maroc, l'action organisée devient visible et se formalise autour des associations de développement et des coopératives laitières, à l'échelle locale, voire régionale. Le lait est aujourd'hui au cœur des dynamiques agraires irriguées au Maroc. Une majorité d'irrigants livrent leur lait à des coopératives et sont concernés par leur fonctionnement et leurs performances. Par la reconquête et l'appropriation des coopératives, les irrigants participent, à des degrés différents, aux réflexions sur l'amélioration de la productivité laitière et investissent les excédents dégagés dans le développement local. Ils ont tendance à transformer les organisations impulsées par l'État en façonnant leurs propres règles, démontrant ainsi la vigueur de l'action organisée, mais répondant parfois très imparfaitement aux attentes de l'État. Ainsi naissent, par exemple, des coopératives laitières de petite taille, proches des communautés mais ayant une masse critique faible et s'intéressant à d'autres domaines que la collecte de lait. Par ailleurs, l'action organisée reste parfois invisible pour l'administration hydraulique quand elle porte sur des objets qui ne concernent pas directement l'activité agricole (eau potable, santé, éducation).

Un apprentissage progressif de l'action organisée se fait alors, comme l'avait remarqué Lanneau (1981, p. 19), en France, concernant l'émergence des coopératives d'utilisation de matériel agricole en commun : «c'est [...] dans la pratique que s'effectue l'apprentissage de la coopération ». Sur la base de pratiques souvent contradictoires, peut s'élaborer dès lors « une attitude coopérative qui [...] réorganise [les pratiques], les intègre, leur donne de nouvelles significations et de nouvelles formes. Nous sommes alors en présence d'un processus d'interstructuration des individus et des institutions » (Lanneau, 1975, p. 34).

Si les dynamiques d'action organisée sont aujourd'hui clairement perceptibles au Maroc, liées à un changement de fond de la professionnalisation agricole, les organisations professionnelles agricoles ne sont pas toujours mobilisées dans les projets de développement rural ni dans la formulation des politiques publiques. Elles auront sans doute à renforcer leurs capacités dans trois domaines (Rondot et Collion, 2001) : (i) des capacités techniques liées à la maîtrise technico-économique de l'objet de l'organisation (lait, eau...) ; (ii) des capacités stratégiques liées à la gestion de l'organisation et à la communication ; (iii) des capacités financières pour drainer et mobiliser des fonds. C'est ainsi que ces organisations pourront prendre leur place dans un dialogue professionnel avec les opérateurs de recherche et de développement et avec les politiques.

Finalement, nous constatons que l'action organisée concerne pour l'instant très peu l'eau du barrage, que ce soit au Maroc ou en Algérie. Tant que l'eau de l'État (Pascon, 1978) restera entre les mains de techniciens dotés de budgets insuffisants et peu enclins à partager un pouvoir et un savoir-faire qu'ils estiment hors de portée des agriculteurs, tant que les agriculteurs trouveront d'autres alternatives, notamment via les arrangements autour de l'eau souterraine, et ne s'investiront pas franchement dans la gestion de l'eau, les aménagements publics péricliteront. En revenant sur la supposée incompatibilité d'une coordination hydraulique centralisée avec des agriculteurs familiaux (Lees, 1986), on peut se demander si l'adaptation doit toujours être du ressort des irrigants, à l'image d'une compatibilité descendante des logiciels. Ne peut-on pas mettre en débat, en même temps, une nécessaire reconfiguration physique et managériale de l'aménagement hydraulique? La possibilité de déléguer la gestion de l'eau de l'État à un opérateur privé, par un partenariat public-privé « décrété », est aujourd'hui envisagée au Maroc. Au-delà des questions idéologiques sur les domaines d'intervention respectifs de l'État et du privé, il est difficile de comprendre qu'un tel partenariat n'implique pas la profession agricole. Au contraire, dans une telle configuration, il nous semble encore plus important que les agriculteurs soient des partenaires reconnus dans les négociations. Il est difficile de leur demander de participer à la mise en œuvre d'un contrat (paiement de redevances, gestion du tour d'eau...) sans que son contenu ait été négocié collectivement. Cependant, comme l'explique Ostrom (1992), leur implication dans l'élaboration du contrat n'est pas suffisante et ne donne pas de garanties pour une application ex post des règles convenues. Mieux vaut intégrer une flexibilité opérationnelle dans la construction institutionnelle, permettant aux différentes parties de faire évoluer les règles en les adaptant à des situations hétérogènes et évolutives. En absence d'une telle construction institutionnelle adaptative, les agriculteurs continueront à recourir à des arrangements "illicites » et à opposer une résistance au moins passive au projet d'irrigation.

\section{Conclusions}

\section{Retour sur l'hypothèse d'une recherche d'autonomie et de renégociation de la dépendance}

Nous avons cherché à appréhender les différentes formes d'action organisée, impliquant principalement les 
irrigants, au sein des périmètres de grande hydraulique au Maroc et en Algérie. Il existe une multitude d'actions, souvent informelles mais permettant aux agriculteurs une adaptation progressive aux changements qui interviennent (retrait des services de l'État, apparition de nouveaux acteurs, accès aux nouvelles ressources). Par ces arrangements, les agriculteurs obtiennent une certaine autonomie par rapport à la coordination centralisée de la grande hydraulique, notamment à travers l'accès aux eaux souterraines.

Les différents cas présentés montrent que des moments de rupture et de rencontre se suivent, mais que le contact entre agriculteurs et services de l'État est rarement rompu, confirmant l'hypothèse d'une motivation portant sur la renégociation de la dépendance. Les rapports pédagogiques ont aussi nettement évolué ; le technicien se positionne, individuellement, de moins en moins comme l'unique détenteur de savoir et cherche des solutions aux demandes formulées. Cependant, ce repositionnement ne se traduit pas explicitement dans les choix et les orientations de l'administration, qui continue à diagnostiquer, analyser, formuler des solutions pour ensuite «sensibiliser » les agriculteurs à leur mise en œuvre. Ces solutions, dans la majorité des cas, ont toute la force de la cohérence technique et de l'efficience économique, mais sont dépourvues d'acceptabilité socioculturelle. Cette faiblesse explique en partie les échecs cumulés de projets collectifs et la méfiance qu'a développée la population rurale vis-à-vis des recommandations qui leur sont faites.

\section{Des implications pour l'action publique}

La vigueur de l'action organisée dans les périmètres de grande hydraulique, formalisée ou non, incite à réfléchir à sa contribution à l'action publique. Le constat d'une décentralisation de compétences et d'initiatives dans le sens de Gaudin (2004) justifie que nous nous soyons d'abord intéressés à l'action organisée des irrigants pour mieux repenser l'action publique. Même en grande hydraulique, elle n'est plus l'apanage de l'État aménageur, mais bien un construit mêlant les initiatives d'une multitude d'acteurs à plusieurs échelles. Il importe alors de comprendre comment et pourquoi s'opère l'action organisée et à quoi elle aboutit, de même que la capacité des irrigants à combler, à travers cette action, des «appels d'air » créés par le redéploiement de l'État. Cette compréhension ne doit pas relever uniquement d'une curiosité scientifique, elle doit aussi fournir les éléments d'une pédagogie de développement centrée davantage sur la fonction d'accompagnement que sur la formation ou, moins encore, sur la sensibilisation. Cette nouvelle praxis interpelle alors la recherche mais aussi le monde du développement agricole.

\section{Remerciements}

Nos travaux se sont déroulés dans le cadre du projet SIRMA, financé par le ministère français des Affaires étrangères et européennes. Nous remercions l'ensemble des acteurs pour leur disponibilité et leurs appuis. Les opinions exprimées sont de la seule responsabilité des auteurs.

\section{Références}

Bessaoud, O., 2006. La stratégie de développement rural en Algérie, Options méditerranéennes, série A, 71, 79-89.

Bouderbala, N., 1977. La formation du système foncier au Maroc, in Bouderbala, N., Chraibi, M., Pascon, P. (Eds), La Question agraire, 2, 151-166.

Caron, P., 1998. Espace, élevage et dynamique du changement : analyse, niveaux d'organisation et action. Le cas du Nordeste semi-aride du Brésil. Thèse de doctorat, Université Paris $\mathrm{X}$, Paris.

Chaulet, C., 1984. La Terre, les frères et l'argent, 3 tomes, Alger, Office des publications universitaires.

Coward, E.W., 1980. Irrigation development: institutional and organizational issues, in Coward, E.W. (Ed.), Irrigation and Agricultural Development in Asia: Perspectives from the Social Sciences, Ithaca and London, Cornell University Press.

Desrues, T., 2005. La politique agricole au Maroc et la question des associations de producteurs agricoles, New Medit, IV, 2, 16-25.

Diemer, G., Slabbers, J., 1992. Irrigators and engineers in developing countries, in Diemer, G., Slabbers, J. (Eds), Irrigators and Engineers, Amsterdam, Thesis Publishers, 3-13.

Egg, J., Deme, M., 2002. La libéralisation du marché du riz, in Kuper, M., Tonneau, J.-P. (Eds), L'Office du Niger, grenier à riz du Mali : succès économiques, transitions culturelles et politiques de développement, Montpellier, Cirad / Paris, Karthala, 88-92.

Friedberg, E., 1993. Le Pouvoir et la règle : dynamiques de l'action organisée, Paris, Le Seuil.

Friedberg, E., 2005. La dynamique de l'action organisée, in Cabin, P., Choc, B. (Eds), Les Organisations : état des savoirs, $2^{\mathrm{e}}$ éd. actualisée, Paris, Éditions Sciences humaines, 41-46.

Gaudin, J.-P., 2004, L'Action publique : sociologie et politique, Paris, Dalloz-Sirey.

Guillermou, Y., 1994. Encadrement étatique et formes d'organisation autonomes en secteur paysan marginalisé : cas de l'Algérie et du Congo, in Jacob, J.-P., Lavigne Delville, P. (Eds), Les Associations paysannes en Afrique : organisation et $d y$ namiques, Marseille, APAD / Paris, Karthala / Genève, IUED, Paris, 201-218.

Hunt, R.C., Hunt, E., 1976. Canal irrigation and local social organization, Current Anthropology, 17, 3, 389-411.

Imache, A., 2008. Construction de la demande en eau agricole au niveau régional en intégrant le comportement des agriculteurs : application aux exploitations agricoles collectives de la Mitidjaouest (Algérie). Thèse de doctorat, AgroParisTech, Paris.

Imache, A., Bouarfa, S., Kuper, M., Hartani, T., Dionnet, M., à paraître. Integrating "invisible" farmers into a regional debate on water productivity: the case of informal water and land markets in the Algerian Mitidja plain, Irrigation and Drainage. 
Jacob, J.-P., Lavigne Delville, P., 1994. Les Associations paysannes en Afrique : organisation et dynamiques, Marseille, APAD / Paris, Karthala / Genève, IUED.

Johnson, S.H., Vermillion, D.L., Sagardoy, J.A., 1995. Irrigation Management Transfer: Selected Papers from the International Conference on Irrigation Management Transfer, Wuhan, China, 20-24 September 1994. Water Report 5 Food and Agriculture Organization of the United Nations and the International Irrigation Management Institute, Rome, Colombo.

Jollivet, M., 1992. Pluridisciplinarité, interdisciplinarité et recherche finalisée ou des rapports entre sciences, techniques et sociétés, in Jollivet, M. (Ed.), Sciences de la nature, sciences de la société : les passeurs de frontières, Paris, CNRS Éditions.

Jouve, A.-M., 1999. Évolution des structures de production et modernisation du secteur agricole au Maghreb, in Jouve, A.-M., Bouderbala, N. (Eds), Politiques foncières et aménagement des structures agricoles dans les pays méditerranéens, Cahiers Options méditerranéennes, 36, 223-233.

Kemmoun, H., Kuper, M., Errahj, M., 2004. La vidéo comme support de concertation autour de la gestion de l'eau d'irrigation en grande hydraulique au Maroc, in Richard-Ferroudji, A., Caron, P., Jamin, J.-Y., Ruf, T. (Eds). Coordinations hydrauliques et justices sociales: actes $d u 4^{e}$ séminaire PCSI, 25-26 novembre 2004, Montpellier, Cirad, CD-ROM.

Lanneau, G., 1975. L'entraide et la coopération au village, in Fabre, D., Lacroix, J. (Eds), Communautés du Sud : contribution à l'anthropologie des collectivités rurales occitanes, Paris, UGE, t. II, 435-499.

Lanneau, G., 1981. L'engagement coopératif : pratiques et attitudes, Revue des études coopératives, 205, 2, 43-73.

Lees, S.H., 1986. Coping with bureaucracy: Survival strategies in irrigated agriculture, American Anthropologist, New Series, $88,3,610-622$.

Reçu le 6 février 2008. Accepté le 5 mars 2009.
Mercoiret, M.-R., 2005. Les agricultures familiales et leur rôle pour un développement durable : choix politiques et enjeux, in Caron, P. (Ed.), Agricultures familiales et mondes à venir, Montpellier, Cirad.

Mesli, M.E., 2007. L'Agronome et la terre, Alger, Alpha.

Mollinga, P., Bolding, A. (Eds), 2004. The Politics of Irrigation Reform: Contested Policy Formulation and Implementation in Asia, Africa and Latin America, Aldershot, Ashgate.

Ostrom, E., 1992. Crafting Institutions for Self-Governing Irrigation Systems, San Francisco, Institute for Contemporary Studies.

Osty, P.-L., 1978. L'exploitation vue comme un système. Diffusion de l'innovation et contribution au développement, BTI, 326, 43-49

Oudin, É., 2006. Analyse et propositions d'améliorations du fonctionnement de coopératives de collecte laitière au Maroc: approche par les systèmes d'information. Mémoire de DEA, INA P-G, Paris.

Pascon, P., 1978. De l'eau du ciel à l'eau de l'État : psychosociologie de l'irrigation, Hommes, terre et eaux, 8, 28, 3-10.

Popp, H., 1984. Effets socio-géographiques de la politique des barrages au Maroc, 2 tomes, Rabat.

Rondot, P., Collion, M.-H., 2001. Organisations paysannes : leur contribution au renforcement des capacités rurales et à la réduction de la pauvreté. Compte rendu des travaux, Washington (DC), 28-30 juin 1999. Département du développement rural, Banque mondiale, Washington (DC).

Shah, T., 1995. Making farmers' Cooperatives Work: Design, Governance and Management, New Delhi, Sage Publications India.

Sraïri, M.T., Ben Salem, M., Bourbouze, A., Elloumi, M., Faye, B., Madani, T., Yakhlef, H., 2007. Analyse comparée de la dynamique de la production laitière dans les pays du Maghreb, Cahiers Agricultures, 16, 4, 251-257. 